\title{
Hypermanganesemia with Dystonia, Polycythemia and Cirrhosis (HMDPC) due to mutation in the SLC30A10 gene.
}

\begin{abstract}
:
Manganese $(\mathrm{Mn})$ is an essential element for metabolic pathways but it can be toxic when present in excessive amounts in the body. Hypermanganesemia along with dystonia, polycythemia, characteristic MRI brain findings in the basal ganglia, and chronic liver disease are the hallmarks of an inherited $\mathrm{Mn}$ transporter defect due to mutations in the SLCBOA10 gene. We are reporting three siblings who presented with features of dystonia, polycythemia, MRI brain showing basal ganglia hyperintensity on T1 weighted images and chronic liver disease. Blood Mn levels were markedly elevated in the affected patients. Mutation analysis of DNA samples of the affected children confirmed a homozygous missense mutation in SLC3OA10. Chelation therapy with intravenous disodium calcium edetate was started in two siblings and led to a marked decrease in whole blood Mn. Oral Penicillamine was later added to the therapy which further improved blood $\mathrm{Mn}$ levels. This is a rare disorder and is one of the potentially treatable inherited metal storage disorders. It can be fatal if left untreated. Penicillamine may be an effective alternative to disodium calcium edetate.
\end{abstract}

Key words: Inherited hypermanganesemia, Polycythemia, SLC30A10 mutation. 
Introduction:

Manganese $(\mathrm{Mn})$ is an essential trace metal that plays a critical role as a cofactor for a variety of enzymes involved in amino acid, lipid and carbohydrate metabolism, immune function, bone and connective tissue growth and blood clotting [1].

Despite its requirement in multiple physiological processes, elevated levels of $\mathrm{Mn}$ trigger toxicity, particularly within the central nervous system (CNS), causing cognitive, psychiatric and motor abnormalities [2].

Mn intoxication can occur in different professionals [3], in individuals with chronic liver disease [4], in prolonged total parenteral nutrition administration [5] and in drug-addicts using Mn contaminated ephedrone peparations [6]. The first inborn error of $\mathrm{Mn}$ metabolism has recently been identified by Tuschl et al [7]. This autosomal recessive disorder, called Hypermanganesemia with Dystonia, Polycythemia and Cirrhosis (HMDPC), is caused by mutations in the SLC3OA10 gene, encoding a Mn transporter, which results in Mn accumulation, mainly in the basal ganglia, cerebellum and liver, and causes a syndrome of early-onset generalized dystonia, cirrhosis, polycythemia, and hypermanganesemia $[7,8]$. The characteristic MRI findings of this syndrome are hyperintense signals in the basal ganglia on T1 weighted and hypointense signals on T2 weighted images. 


\section{Case reports:}

We are reporting a case series of three siblings with childhood onset dystonia. Parents have a consanguineous marriage (first cousins) and have twelve children. The index case is 13 years old; the other affected siblings are 23 years and 8 years old. This family belongs to Sind, Pakistan. They consume tap water, the analysis of which yielded normal Mn levels.

\section{Patient 1}

The index case was a previously healthy 13 year old male with history of difficulty in walking which started at the age of 10 years and progressively worsened. Slurred speech was present from two years of age. In addition, difficulty in swallowing had developed recently. His birth history was uneventful. He achieved all milestones appropriate for his age. He went to school up until 10 years of age and had average performance in his studies. On general physical examination, his palms and conjunctivae were erythematous. Higher mental functions were intact and speech was dysarthric. Motor examination showed marked dystonia of his limbs which was elicited during walking. There was no ataxia, tremors or cranial nerve involvement and systemic examination was unremarkable. Initially, a differential diagnosis of Wilson's disease, Hallervorden-Spatz disease and hereditary dystonia was made. Initial work up was remarkable for polycythemia while 24 hour urinary copper was normal (Table 1). MRI brain showed T1 hyperintensity of globus pallidus, caudate nucleus, midbrain and tectum with concurrent hypointensity on T2 weighted images (Fig. 1). This suggested the possibility of iron or $\mathrm{Mn}$ deposition in the brain. Whole blood Mn was markedly raised at $14972.6 \mathrm{nmol} / \mathrm{L}$ (normal $<320 \mathrm{nmol} / \mathrm{L}$ ) suggesting a diagnosis of HMDPC. An ultrasound abdomen was done which showed no evidence of chronic liver disease or cirrhosis. SLC30A10 mutation analysis was performed, which was positive for a homozygous missense mutation in the 4th exon of the SLC30A10 gene (c.1006C $>$ T; p. H336Y) (Fig 2). The child was started on disodium calcium edetate infusions at a dose of $1 \mathrm{gm} / \mathrm{m} 2$ for 5 days every 6 weeks, and oral iron was also given. After 5 cycles of disodium calcium edetate infusions, blood Mn level had decreased to 1777.7 $\mathrm{nmol} / \mathrm{L}$. Parents could not afford any further disodium calcium edetate, hence, a trial of Penicillamine was started and the Mn level was repeated after 6 months of therapy. There was a good response to Penicillamine noted as his $\mathrm{Mn}$ level had further declined to $1092 \mathrm{nmol} / \mathrm{L}$. In addition he also showed clinical improvement in his overall motor activity. He was more stable in his walking and falling less and his dysphagia had improved. 


\section{Patient 2}

This previously healthy 8 year old boy started to have difficulty in speech for 6 months and difficulty in walking for 3 months. On examination he was active and alert. He had dysarthria and motor examination revealed significant dystonia of lower limbs during walking which resulted in tip toeing and subsequently difficulty in walking. There was no ataxia or tremors.

Initial work up was also remarkable for polycythemia and high whole blood manganese levels (Table1). MRI brain showed the same findings as the index case. This child had evidence of chronic liver disease on ultrasound only. Genetic evaluation confirmed the same homozygous mutation in the SLC30A10 gene (Fig 2). Treatment was started with disodium calcium edetate infusion and oral iron supplements. After 4 cycles repeat blood $\mathrm{Mn}$ level had decreased (Table 1). Subsequently, Penicillamine was started and repeat $\mathrm{Mn}$ level was done after 6 months. There was a good response to Penicillamine and his Mn level decreased significantly, along with clinical improvement in dystonia.

\section{Patient 3}

This 23 year old male was well up to the age of 6 years when he started having difficulty in walking and speaking which was followed by frequent falls. He eventually became wheel chair bound within a few months. On examination he had dysarthria but his cognitive functions were good. He had increased tone, dystonia of limbs and contractures of lower limbs with no cranial nerve involvement. He had no involuntary movements. His laboratory work up was also remarkable for polycythemia and high whole blood Mn level (Table 1). The MRI brain showed similar changes in the basal ganglia as the index case. Genetic evaluation confirmed the homozygous mutation in the SLC3OA10 gene. He has also been recently started on oral penicillamine and iron supplements.

The father and one other unaffected sibling were shown to be heterozygous for the p. H336Y mutation. Genetic testing for the rest of the family could not be done as DNA samples were not available (Fig 2). The mutation identified has been reported in heterozygous state in one South Asian individual with an allele frequency of 1:8252 on the ExAC database (http://exac.broadinstitute.org). 


\section{Discussion:}

SLC30A10 is a crucial Mn transporter in man. In individuals with homozygous SLC30A10 mutations, hepatic $\mathrm{Mn}$ excretion is severely defective, leading to $\mathrm{Mn}$ accumulation in the liver, the bloodstream, the brain and other peripheral tissues [9]. Mn induces erythropoietin gene expression leading to polycythemia.

In all three patients symptoms started before 10 years of age and common presentation was difficulty in walking, dysarthria and dystonia. The index case presented at 13 years of age and after his diagnosis was established the parents brought the other two siblings for evaluation. The younger sibling was 6 years at presentation and he had only very mild symptoms while the older sibling was already wheel chair bound. All had polycythemia on presentation while hepatic dysfunction was only seen in patient 2 who had evidence of chronic liver disease. Quadri et al also report that hepatic dysfunction may vary between members of the same family $[7,8]$.

Some intrafamilial clinical heterogeneity was noted amongst our children and this has been observed by Delnooz et al. The characteristic MRI findings of this syndrome are hyperintense signals in the basal ganglia on T1 weighted images and hypointense signals on T2 weighted images. The reverse is seen in copper deposition in Wilson's disease which shows hypointense signals on T1 weighted images and hyperintense signals on T2 weighted images in the basal ganglia. The diagnosis of this rare syndrome is confirmed by identification of bi-allelic mutations in the SLC30A10 gene. SLC3OA10 has recently been identified as the disease causing gene by Tuschl et al and four Pakistani siblings were amongst the initial group reported [10].

The mainstay of treatment is chelation therapy. Chelation therapy with disodium calcium edetate (CaNa2-EDTA $1 \mathrm{~g} / \mathrm{m}^{2}$ daily) [11] enhances urinary $\mathrm{Mn}$ excretion and significantly improves neurological symptoms [7].

Other chelating agents have been used including oral dimercaptosuccinic acid (DMSA) and Paraaminosalicylic acid [12]. In our patients only two were started on disodium calcium edetate.

The role of Penicillamine has not been evaluated extensively in the treatment of this disorder. We used Penicillamine which showed an excellent response in declining blood $\mathrm{Mn}$ levels and a relatively good 
clinical response in both patients. There is a possibility that Penicillamine may be used in the treatment of hypermanganesemia, however long term efficacy of this drug in this disorder remains to be evaluated.

\section{Conclusion:}

We wish to highlight this rare disorder which, together with Wilson's disease, is the only potentially treatable inherited metal storage disorder to date. The role of Penicillamine as an effective Mn chelator needs to be further evaluated. 


\section{References:}

[1] Aschner M, Guilarte TR, Schneider JS, Zheng W. Manganese: recent advances in understanding its transport and neurotoxicity. Toxicol Appl Pharmacol 2007; 221:131-47.

[2] Dobson A, Erikson K, Aschner M. Manganese Neurotoxicity. Ann NY Acad Sci 2004; 1012: 115-28.

[3] Huang CC, Chu NS, Lu CS, Wang JD, Tsai JL, Tzeng JL, et al. Chronic manganese intoxication.

Arch Neurol 1989; 46:1104-6.

[4] Butterworth RF. Metal toxicity, liver disease and neurodegeneration. Neurotox Res2010; 18:100-5.

[5] Chalela JA, Bonillha L, Neyens R, Hays A. Manganese encephalopathy: An under-recognized condition in the intensive care unit. Neurocrit Care 2011; 14:456-8.

[6] Sanotsky Y, Lesyk R, Fedoryshyn L, Komnatska I, Matviyenko Y, Fahn S. Manganic encephalopathy due to "ephedrone" abuse. Mov Disord 2007; 22:1337-43.

[7] Tuschl K, Mills PB, Parsons H, Malone M, Fowler D, Bitner-Glindzicz M, et al. Hepatic cirrhosis, dystonia, polycythaemia and hypermanganesaemia-a new metabolic disorder. J inherit Metab Dis 2008; 31:151-63.

[8] Quadri M, Federico A, Zhao T, Breedveld GJ, Battisti C, Delnooz C, et al. Mutations in SLC3OA10 cause parkinsonism and dystonia with hypermanganesemia, polycythemia, and chronic liver disease. Am J Hum Genet 2012; 90:467-77.

[9] Sreedharan S, Stephansson O, Schiöth HB, Fredriksson R. Long evolutionary conservation and considerable tissue specificity of several atypical solute carrier transporters. Gene $2011 ; 478: 11-8$.

[10] Tuschl K, Clayton PT, Gospe SM, Gulab S, Ibrahim S, Singhi P, et al. Syndrome of hepatic cirrhosis, dystonia, polycythemia, and hypermanganesemia caused by mutations in SLC30A10, a manganese transporter in man. Am J Hum Genet 2012; 90:457-66.

[11] Hernandez EH, Discalzi G, Valentini C, Venturi F, Chio A, Carmellino C, et al. Follow-up of patients affected by manganese-induced Parkinsonism after treatment with CaNa 2 EDTA. Neurotoxicology 2006; 27:333-9. 
[12] Brna P, Gordon K, Dooley JM, Price V. Manganese toxicity in a child with iron deficiency and polycythemia. J Child Neurol 2011; 26:891-4.

Page $\mid 8$ 
Table1:

Clinical and laboratory findings of patients with Hypermanganesemia, Polycythemia, Dystonia and Cirrhosis.

\begin{tabular}{|c|c|c|c|}
\hline & Case 1 & Case 2 & Case 3 \\
\hline Gender & Male & Male & Male \\
\hline Age of patient & 13 yrs & $8 y r s$ & 23 yrs \\
\hline Dystonia & Present & Present & Present \\
\hline Dysarthria & Present & Present & Present \\
\hline Hepatomegaly & No & No & No \\
\hline Hemoglobin *(13-16g/dl) & $20.7 \mathrm{~g} / \mathrm{dl}$ & $18.3 \mathrm{gm} / \mathrm{dl}$ & $16.4 \mathrm{gm} / \mathrm{dl}$ \\
\hline Hematocrit *(42-16\%) & $61.7 \%$ & $53.5 \%$ & $47.6 \%$ \\
\hline $\mathrm{SGPT} *(<45 \mathrm{IU} / \mathrm{L})$ & 43IU/L & $44 \mathrm{IU} / \mathrm{L}$ & $381 \mathrm{U} / \mathrm{L}$ \\
\hline Ferritin levels & $13.2 \mathrm{ng} / \mathrm{ml}$ & -- & -- \\
\hline \multicolumn{4}{|l|}{$*(7-140 \mathrm{ng} / \mathrm{ml})$} \\
\hline 24hrs urinary Copper & 18ug/day & -- & -- \\
\hline \multicolumn{4}{|l|}{ *(<60ug/day) } \\
\hline Erythropoietin & 18.55 & -- & -- \\
\hline \multicolumn{4}{|l|}{ *(3.3-16.6 U/m) } \\
\hline Blood Mn level & $14972.6 \mathrm{nmol} / \mathrm{L}$ & $1511 \mathrm{nmol} / \mathrm{L}$ & $539 \mathrm{nmol} / \mathrm{L}$ \\
\hline \multicolumn{4}{|l|}{$*(<320 \mathrm{nmol} / \mathrm{L})$} \\
\hline Blood Mn levels after 6 months & $1777.7 \mathrm{nmol} / \mathrm{L}$ & $1481.4 \mathrm{nmol} / \mathrm{L}$ & -- \\
\hline Ultrasound liver & Normal & Chronic liver disease & Normal \\
\hline MRI brain suggestive of & Yes & Yes & Yes \\
\hline \multicolumn{4}{|l|}{ Mn deposition } \\
\hline SLC30A10 mutations & Positive & Positive & Positive \\
\hline
\end{tabular}


-- not done,

*normal range

Page | 10 
Figure 1

MRI brain axial view of Index case

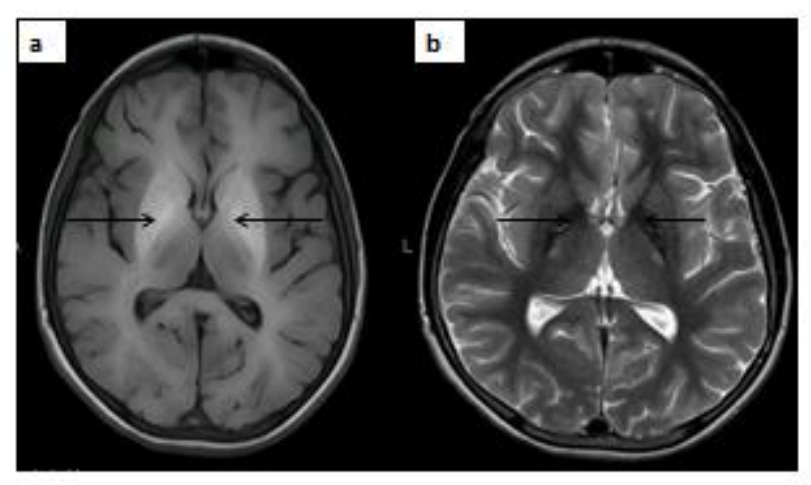

1(a). Axial view of Index case, arrows indicating bilateral hyperintense signals on TW1 image in basal ganglia along with few cystic changes in this region.

1(b). Bilateral Hypointense signals on TW2 image in basal ganglia. 
Figure 2

Pedigree and sequencing analysis of SLC30A10

2(a)

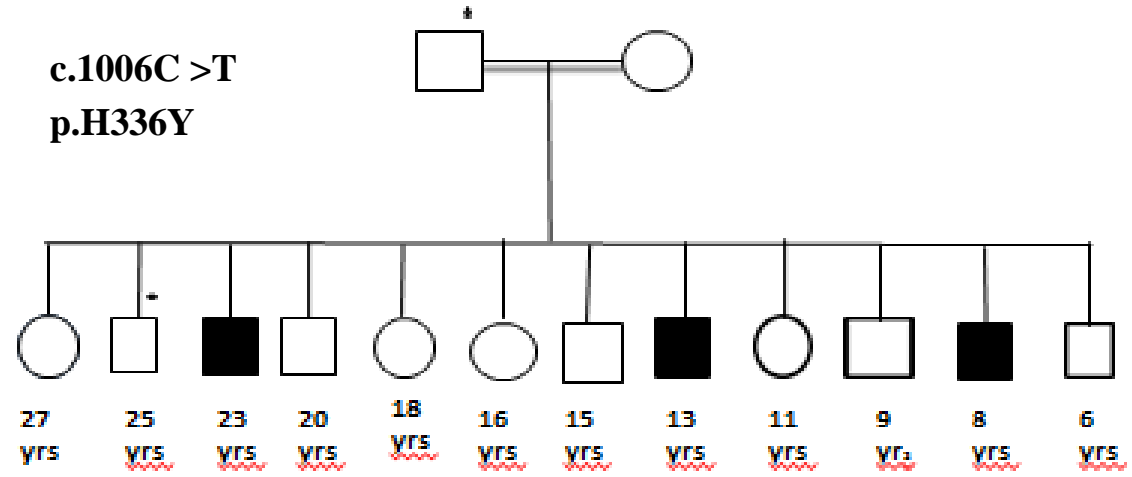

2(b)

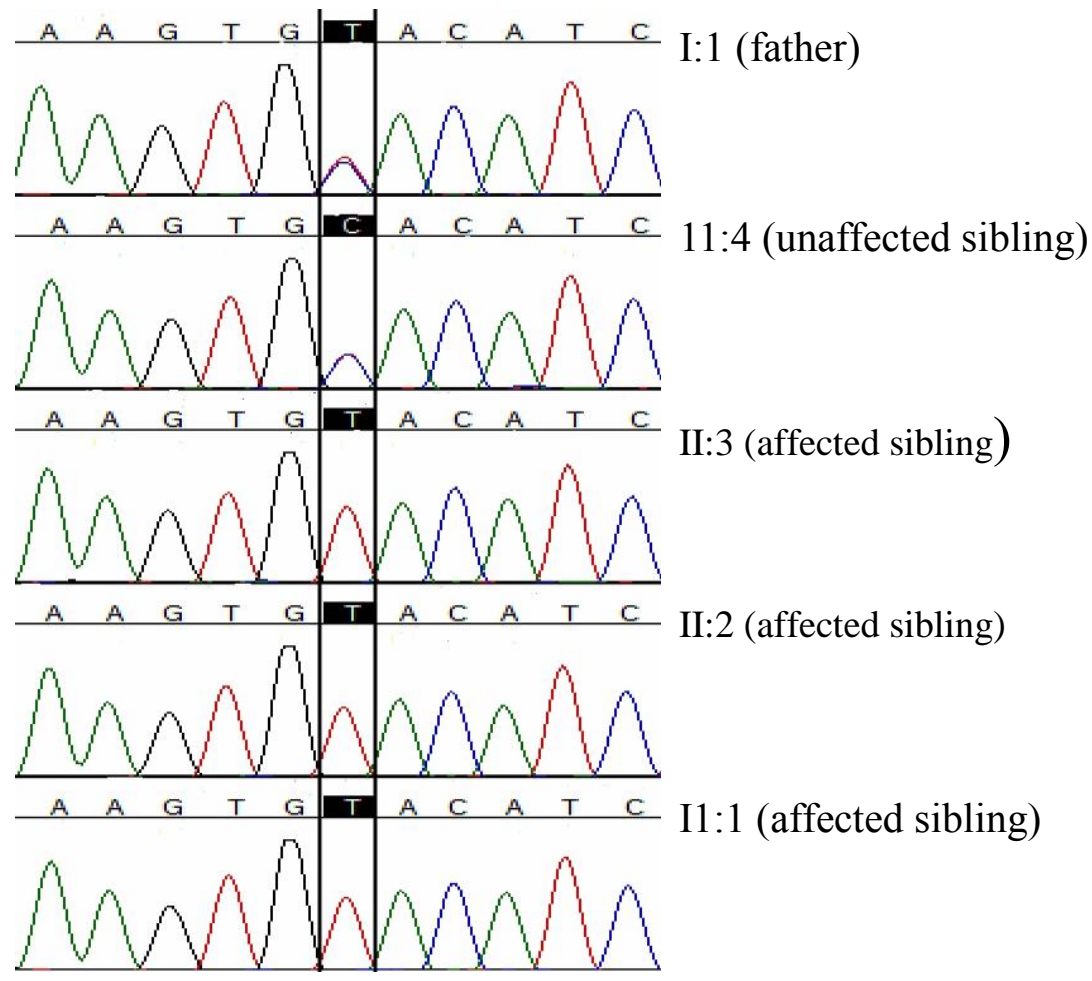

2(a). Pedigree of the described family: Affected individuals are indicated by black shading. 2(b). Sequence chromatograms showing a homozygous missense change (c.1006C $>T$ ) in three affected siblings (II:1, II:2 and II:3). Sequence analysis of DNA from the father (I:1) and an unaffected sibling (II:4) confirms that they are heterozygous carriers for the identified mutation. DNA from the mother and the remaining siblings was unavailable. Mutated bases are boxed in black. 